\title{
A comparative study of azelastine, cromolyn and olopatadine ophthalmic solution in vernal keratoconjunctivitis in a tertiary care hospital-open label parallel group study design
}

\author{
Gaurav Sharma ${ }^{1}$, Kulbhushan P. Chaudhary ${ }^{3}$, Sushma Sawaraj ${ }^{2 *}$
}

\author{
${ }^{1}$ Department of Ophthalmology, ${ }^{2}$ Department of Pharmacology, Dr. RPGMC Tanda Kangra, Himachal Pradesh, India \\ ${ }^{3}$ Department of Ophthalmology, IGMC Shimla (Rtd), Himachal Pradesh, India
}

Received: 21 April 2021

Revised: 20 May 2021

Accepted: 25 May 2021

\section{*Correspondence:}

Dr. Sushma Sawaraj,

Email: sushmaswaraj07@gmail.com

Copyright: (C) the author(s), publisher and licensee Medip Academy. This is an open-access article distributed under the terms of the Creative Commons Attribution Non-Commercial License, which permits unrestricted non-commercial use, distribution, and reproduction in any medium, provided the original work is properly cited.

\begin{abstract}
Background: Vernal keratoconjunctivitis (VKC) is a chronic, bilateral, external ocular inflammatory disease primarily affecting young boys living in warm, dry climates with seasonal variations. The disease causes lot of discomfort to the patient and sometimes can predispose to serious problems like shield ulceration and keratoconus. A number of drugs are used in the management of the condition, with variable results. The aims and objectives of this study was to compare the efficacy and safety of the drugs, cromolyn sodium, azelastine and olopatadine ophthalmic solutions in the treatment of VKC.

Methods: Sixty patients of VKC were studied over a period of 6 weeks. They were divided into 3 groups randomly to receive one of the drugs under study. Symptoms and signs were recorded after detailed questioning and examination according to modified criterion of Tabbara and Arafat.

Results: There was significant reduction in the mean itching scores with olopatadine as compared to cromolyn sodium and azelastine $(\mathrm{p}<0.05)$. Olopatadine significantly decreased mean lacrimation scores as compared to cromolyn sodium and azelastine $(\mathrm{p}<0.005)$. Olopatadine, cromolyn and azelastine showed significant reduction of corneal stippling, but no drug was significantly better than the other. Both cromolyn and olopatadine showed reduction of limbal edema equally $(\mathrm{p}<0.05)$, olopatadine reduced limbal edema more significantly as compared to azelastine $(\mathrm{p}<0.05)$.

Conclusions: All the three drugs were found to be safe in the treatment of VKC. Olopatadine may be preferred over the other two drugs since it reduced both itching and discharge most significantly.
\end{abstract}

Keywords: VKC, Olopatadine, Azelastine, Cromolyn sodium, Allergic conjunctivitis

\section{INTRODUCTION}

VKC is a chronic, bilateral, external ocular inflammatory disease primarily affecting children and adolescents in warm, dry climates with seasonal variations. It is most frequently seen in boys and tends to resolve at puberty. ${ }^{1}$ The disease causes lot of discomfort to the patient and sometimes can predispose to serious complications like shield ulceration and keratoconus involving various corneal components. Chronic inflammation can cause severe visual complications. VKC is more frequent in warmer, arid, windy climates, within the Mediterranean area, Central African Republic, Japan, India and South America but is also reported in North America, China, Australia, Great Britain and Sweden. VKC appears mainly seasonally but can be perennial, with acute exacerbations or chronic. The etiology involves a variety of components, including environmental allergens, climate and genetic 
predisposition. Diagnosing and treating VKC has been a challenge for ophthalmologists, since the pathogenesis is unclear and anti-allergic therapy often unsuccessful. Treatment of VKC requires an all-round approach that includes conservative measures and pharmacologic treatment. ${ }^{2-6}$

A number of drugs are used in the management of the condition with variable results. Mast cell stabilizers are the first-line therapy for VKC. Topical mast cell stabilizers are generally safe and have least ocular side effects, although they may cause some tolerability issues, since transient burning or stinging may occur upon application. The recommended dosing schedule is 2-3 times daily, with a loading period of minimum 7 days and an onset of activity may take as much as 2 weeks. Monotheraphy with disodium cromoglycate (DSCG ) has limited effects in the treatment of $\mathrm{VKC}$ and is less well tolerated than newer anti-allergic compounds. The present study was undertaken to compare efficacy and safety of olopatadine with azelastine and cromolyn ophthalmic solution in the management of the condition.

\section{Aims and objectives}

The aims and objectives of the study was to compare the efficacy and safety of the drugs cromolyn sodium, azelastine and olopatadine ophthalmic solutions in the treatment of VKC.

\section{METHODS}

It was a prospective, open label, parallel group, single center study designed to compare the efficacy and safety of the drugs cromolyn sodium, azelastine and olopatadine ophthalmic solutions in the treatment of VKC. It was conducted in a tertiary care hospital (medical college) in Himachal Pradesh for a period of 1 year. The study was started after obtaining prior approval of the institutional protocol and institutional ethics committee. Written informed consent was obtained from each participant before recruitment. All male and female patients with VKC visiting the ophthalmology outpatient department (OPD) of a tertiary care hospital in Himachal Pradesh were screened. Sixty patients fulfilling the inclusion criteria of VKC were enrolled and studied over a period of 6 weeks. They were divided into 3 groups randomly. Group A received olopatadine ophthalmic solution, group B received cromolyn ophthalmic solution, group $C$ received azelastine ophthalmic solution.

\section{Inclusion criteria}

All patients diagnosed as having VKC clinically were included in the study.

\section{Exclusion criteria}

Patients treated systemically or topically within one month with steroids, infectious diseases as trachoma, bacterial conjunctivitis and staphylococcal blepharitis, patients receiving systemic treatment for associated systemic diseases as asthma and patients receiving subtenon or subconjunctival corticosteroid injection were excluded from the study.

Symptoms and signs were recorded after detailed questioning and examination according to modified criterion of Tabbara and Arafat. ${ }^{7}$ Follow up was done weekly for 6 weeks and grading of signs and symptoms done at each week. Grading of ocular symptoms and signs was done according to modified criterion of Tabbara and Arafat. $^{7}$ Of these we had taken six parameters three symptoms and three signs, that is, photophobia, itching, lacrimation, pupillae, superficial corneal strippling and limbal edema and scores of all patients before treatment and following treatment were recorded. The disease severity was graded from total scores thus obtained as mild $=0-6, \quad$ moderate $=7-12, \quad$ severe $=13-18$. Statistical analysis was done using Chi square test for discreet variables and t test of independent variables. $P$ value was calculated for all variables. A p value $<0.05$ was taken as significant and a p value $>0.05$ was taken as insignificant.

\section{RESULTS}

Out of 60 patients, 48 were males and 12 were females. Age distribution of the patients was 5-10 years (38), 11-15 years (8), 16-20 years (14). Mixed (22), bulbar (24), palpebral (14). Photophobia was decreased significantly by olopatadine, cromolyn and azelastine $(\mathrm{p}<0.005)$. There was significant reduction in the mean itching scores with olopatadine as compared to cromolyn sodium and azelastine $(\mathrm{p}<0.05)$ (Figure 1).

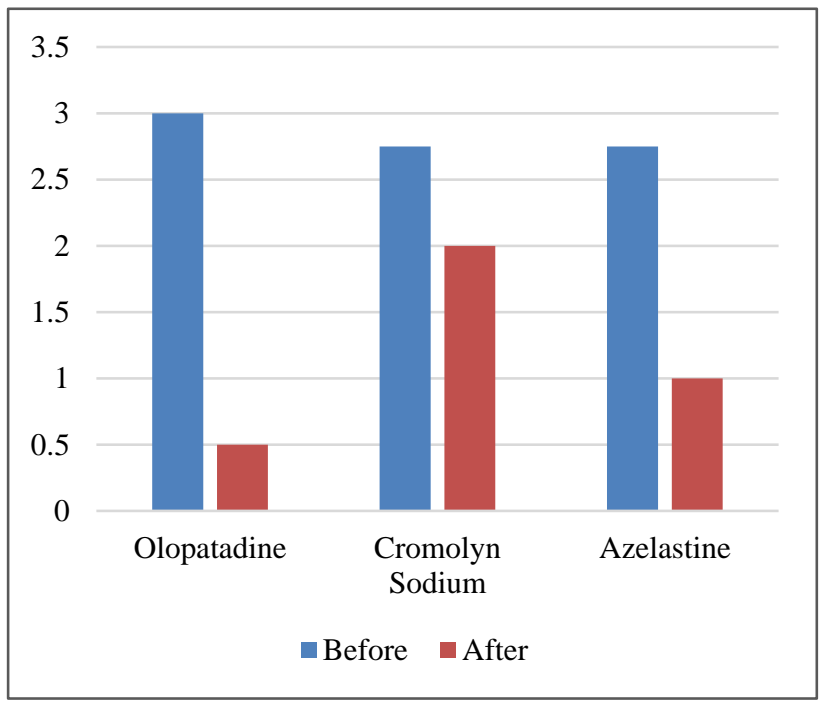

Figure 1: Mean itching score.

Olopatadine significantly decreased mean lacrimation scores as compared to cromolyn sodium and azelastine $(\mathrm{p}<0.005)$ (Figure 2). Azelastine showed better control of papillae as compared to olopatadine. Olopatadine, cromolyn and azelastine showed significant reduction of 
corneal stippling, but there no drug was significantly better than the other. Both cromolyn and olopatadine showed reduction of limbal edema equally $(\mathrm{p}<0.05)$. Olopatadine reduced limbal edema more significantly as compared to azelastine $(\mathrm{p}<0.05)$.

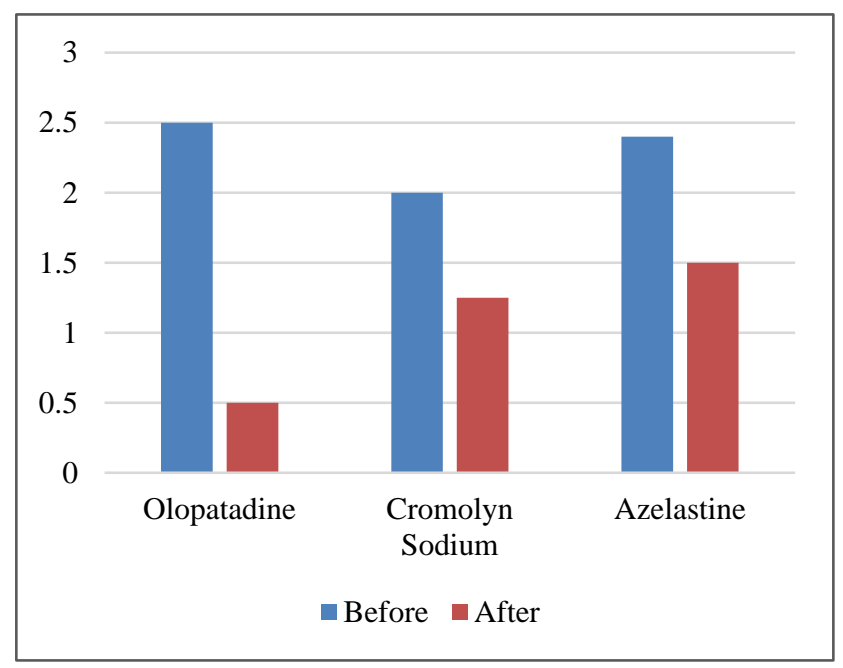

Figure 2: Mean lacrimation score.

\section{DISCUSSION}

VKC is a disease which not only causes a lot of discomfort with minimal interference with day today life but can also predispose patient to serious complications like keratoconus and shield ulcerations and other sight threatening complications.

Although a number of agents have been used in past, they have not been able to deal with all its signs and symptoms effectively. Steroids can produce dramatic relief, but chronic and injudicious use has well documented complications. With all this in context this study was conducted to compare the efficacy and safety of mast cell stabilizer olopatadine hydrochloride $0.1 \%$ with two already widely used drugs namely, cromolyn sodium $4 \%$ and azelastine ophthalmic solution $0.05 \%$ in the management of VKC. In our study we found that male patients outnumbered the females in agreement with the work of Neumann and co-workers who found that VKC is a disease predominantly affecting young boys living in warm dry climates. Kawuma, however who studied the clinical picture of $\mathrm{VKC}$ in Uganda found that the disease was equally divided among males and females. Limbal type was more common type of VKC in our study. Limbal type was more common type according to him. ${ }^{8,9}$

We found that cromolyn was least effective than the other two drugs in reducing papillae in VKC. It was less effective than olopatadine in reducing itching and discharge but more effective in reducing limbal edema which may be due to its more potent mast cell stabilizing effect and absence of antihistaminic effect. Chaudhary studied the effects of combined systemic aspirin and cromolyn sodium in intractable VKC and found them to be effective in reducing all the signs and symptoms of the disease. ${ }^{10}$ Katelaris compared the efficacy and safety of cromolyn sodium with olopatadine and found that the later was more efficacious in reducing symptoms and signs of the disease, which was similar to our findings as itching and discharge were more significantly reduced by olopatadine that cromolyn, however cromolyn was more effective in reducing limbal edema according to our study. ${ }^{11}$ Splangler compared the efficacy of olopatadine with azelastine and found that the former was more effective in reducing itching than the later. ${ }^{12}$ This was in agreement with our findings; although azelastine was more effective than olopatadine in reducing papillae in cases of papillary VKC. It was found in our study that discharge was most significantly reduced by olopatadine than both cromolyn and azelastine which may be explained by this fact that olopatadine reduces goblet cell density. Corum studied the effect of olopatadine on goblet cell density and found that it reduces the number of goblet cells. ${ }^{12}$ In our study it was found that olopatadine was well tolerated by children and side effects were mild and none reported significant discomfort on use of the drug. ${ }^{13}$ Lichtenstein studied the safety of olopatadine in children and adolescents and found that it was safe and well tolerated by them. ${ }^{14}$ Olopatadine has an $\mathrm{H} 1$ antihistaminic effect and has mast cell stabilizing effect hence it has a combined effect thus requires no other drug combination as is required with cromolyn sodium thereby minimizing number of drugs required, cost and inconvenience to the patients.

\section{Limitations}

The main limitation of the present study was a relatively smaller sample size, no blinding was done and shorter duration of follow up of the patients. Further studies in which a larger group can be followed up for a longer duration of time may help yield more data.

\section{CONCLUSION}

All the three drugs were found to be safe in the treatment of VKC. There were no serious side-effects reported for any of the three drugs. All drugs were found to be effective in reducing signs and symptoms of VKC. Any of these drugs should be used as first line treatment in VKC since they are devoid of serious side-effects besides being effective in controlling symptoms and signs. Olopatadine may be preferred over the other two drugs since it reduced both itching and discharge most significantly. Olopatadine may also be preferred since it is expected to have better compliance due to twice a day dosage, which has greater significance since the disease primarily affects school going children and will lead to better compliance. In case of predominantly papillary disease Azelastine may be used as a first line agent since it had greatest effect than the other two drugs. Olopatadine may help in reducing the need of steroids and therefore decreasing the incidence of serious side-effects like cataract and glaucoma in VKC patients. There is needed for further research to develop agents that 
could ultimately remove the need for steroids in these patients.

\section{Funding: No funding sources}

Conflict of interest: None declared

Ethical approval: The study was approved by the Institutional Ethics Committee

\section{REFERENCES}

1. Neumann E, Gutmann MJ, Blumankrantz N, Michaelson IC. A review of four hundred cases of vernal conjunctivitis. Am J Ophthalmol. 1959;47(2):166-72.

2. Leonardi A, Sathe S, Bortolotti M, Beaton A, Sack R. Cytokines, matrix metalloproteases, angiogenic and growth factors in tears of normal subjects and vernal keratoconjunctivitis patients. Allergy. 2009;64(5):710-7.

3. Leonardi A, Bogacka E, Fauquert JL, Kowalski ML, Groblewska A, Jedrzejczak-Czechowicz M, et al. Ocular allergy: recognizing and diagnosing hypersensitivity disorders of the ocular surface. Allergy. 2012;67(11):1327-37.

4. Leonardi A. Vernal keratoconjunctivitis: pathogenesis and treatment. Prog Retin Eye Res. 2002;21(3):319. 39.

5. Tabbara KF. Ocular complications of vernal keratoconjunctivitis. Can J Ophthalmol. 1999;34(2):88-92.

6. Bonini S, Lambiase A, Marchi S, Pasqualetti P, Zuccaro O, Rama P, et al. Vernal keratoconjunctivitis revisited: a case series of 195 patients with long-term follow up. Ophthalmology. 2000;107(6):1157-63.

7. Tabbara FK, Arafat TN. Cromolyn effects on vernal keratoconjunctivitis in children. Arch Ophthalmol. 1977;95(12):2184-6.
8. Leonardi A. Management of vernal keratoconjunctivitis. Ophthalmol Ther. 2013;2(2):7388 .

9. Kawuma M. The clinical picture of vernal keratoconjunctivitis in Uganda. Commun Eye Health. 2001;14(40):66-7.

10. Chaudhary KP. Evaluation of combined systemic aspirn and cromolyn sodium in intractable vernal catarrh. Ann Ophthalmol. 1990;22(8):314-8.

11. Katelaris CH, Ciprandi G, Missoten L, Turner FD, Bertin D, Berdeaux G, et al. A comparison of efficacy and tolerability of olopatadine hydrochloride $0.1 \%$ ophthalmic solution and cromolyn sodium $0.2 \%$ ophthalmic solution in seasonal allergic conjunctivitis. Clin Ther. 2002;24(10):1561-75.

12. Spangler DL, Bensch G, Berdy GJ. Evaluation of clinical efficacy of olopatadine $0.1 \%$ ophthalmic solution and azelastine hydrochloride $0.05 \%$ ophthalmic solution in the conjunctival allergen challenge model. Clin Ther. 2001;23(8):1272-80.

13. Corum I, Yeniad B, Bilgin LK, Ilhan R. Efficiency of olopatadine hydrochloride $0.1 \%$ in the treatment of vernal keratoconjunctivitis and goblet cell density. J Ocul Pharmacol Ther. 2005;21(5):400-5.

14. Lichtenstein SJ, Pasquine TA, Edwards MR, Wells DT, Gross RD, Robertson SM. Safety and tolerability of olopatadine $0.2 \%$ in children and adolescents. $\mathrm{J}$ Ocul Pharmacol Ther. 2007;23(4):366-71.

Cite this article as: Sharma G, Chaudhary KP, Sawaraj S. A comparative study of azelastine, cromolyn and olopatadine ophthalmic solution in vernal keratoconjunctivitis in a tertiary care hospitalopen label parallel group study design. Int J Basic Clin Pharmacol 2021;10:785-8. 